\section{Temperature-induced Glucosinolate Accumulation Is Associated with Expression of BrMYB Transcription Factors}

\author{
Veronica L. Justen \\ Department of Plant and Earth Sciences, University of Wisconsin-River \\ Falls, 324 Agriculture Science, River Falls, WI 54022
}

Vincent A. Fritz ${ }^{1}$

Southern Research and Outreach Center, University of Minnesota, 35838 120th Street, Waseca, MN 56093; and the Department of Horticultural Science, University of Minnesota, 1970 Folwell Avenue, St. Paul, MN 55108

Additional index words. Brassica rapa, chemoprevention, isothiocyanates, quantitative PCR, turnip, thioglucosides

\begin{abstract}
Turnips (Brassica rapa. subsp. rapa L.) produce glucosinolates (GSLs), thioglucosides whose hydrolyzed derivatives have been shown to provide chemopreventive benefits. Two cultivars of turnips ['Just Right' (JR) and 'Scarlet Queen' (SQ)] were grown under three different temperature regimes to assess the role of temperature on GSL production in roots and shoots. When compared with low-temperature treatments, high-temperature treatments increased total and individual GSLs in a tissue- and genotype-specific manner. When compared with low-temperature treatments, total GSLs were $\approx 70 \%$ and $130 \%$ higher in JR shoots and roots, respectively, grown at high-temperature treatments. High temperatures also increased total GSLs in SQ shoots and roots by $\approx 80 \%$ and $85 \%$, respectively, when compared with low temperatures. Gluconasturtiin (GNS, 2-phenylethyl GSL) concentration was inversely correlated with temperature with high-temperature treatments resulting in $20 \%$ and $48 \%$ less GNS than low-temperature treatments in JR and SQ roots, respectively. The indolic GSL, 1-methoxyglucobrassicin (1MGB; 1-methoxy-3-ylmethyl GSL), was the root GSL most elevated by increased temperature resulting in a $1000 \%$ increase on average in both cultivars between the low- and high-temperature treatments. These results show promise for the use of temperature to enhance the health-promoting properties of turnip because 1MGB has potent chemopreventive effects. Gene expression analysis suggests that some BrMYB transcription factor expression levels are associated with temperaturedependent changes in GSL accumulation; however, this association varies between cultivar and tissue type.
\end{abstract}

Glucosinolates are thioglucosides found in Brassica vegetables that when hydrolyzed at the S-glucose bond create a suite of products involved in plant defense, flavor, and human health. Consumption of vegetables containing glucosinolates has been correlated with cancer and disease prevention (Hecht, 2000; Talalay and Fahey, 2001) Glucosinolates and their hydrolyzed derivatives, mainly isothiocyanates, have been shown to serve as chemopreventive agents by stimulating both protection from and excretion of carcinogens and by inhibiting tumor growth (Hecht, 2000; Neave et al., 2005). Identifying vegetable production systems that enhance GSL

\footnotetext{
Received for publication 30 Aug. 2012. Accepted for publication 15 Nov. 2012.

We thank Dr. Adrian Hegeman for the use of his UPLC-MS and Doug Brinkman for technical support.

${ }^{1}$ To whom reprint requests should be addressed; e-mailvafritz@umn.edu.
}

concentrations could have a significant impact on human health, particularly cancer prevention.

GSL concentration is highly influenced by genotype and environmental factors including soil fertility, temperature, and light (Antonious et al., 1996; Charron and Sams, 2004; Engelen-Eigles et al., 2006; Rosen et al., 2005). We found strong correlations between GSL concentrations and temperature in field experiments using colored plastic mulches (Justen et al., 2011). Our previous research using colored plastic mulches in a field setting demonstrated that plastic mulches both alter the light reflected into the canopy of turnip plants and also appreciably change soil temperature (Justen et al., 2011). Depending on the planting date and type of mulch, average soil temperatures under mulches during the turnip growing season can be up to $5{ }^{\circ} \mathrm{C}$ above and $4{ }^{\circ} \mathrm{C}$ below bare soil temperatures (Justen et al., 2011). Temperature can have a significant impact on both shoot and root GSL concentration in a controlled environment. Charron and colleagues found that the concentration of the aliphatic glucosinolate gluconapin (GNP; 3-butenyl GSL) doubled in leaves and stems of B. oleracea plants grown at $32{ }^{\circ} \mathrm{C}$ and the indolic glucosinolate $1 \mathrm{MGB}$ (neoglucobrassicin; 1-methoxy-3-ylmethyl GSL) increased 3 -fold in roots grown at $32{ }^{\circ} \mathrm{C}$ compared with those grown at $12{ }^{\circ} \mathrm{C}$ (Charron and Sams, 2004). Engelen-Eigles and colleagues (2006) found that the concentration of the aromatic glucosinolate gluconasturtiin was increased in Nasturtium officinale grown at 10 and $15^{\circ} \mathrm{C}$ as compared with that grown at 20 or $25^{\circ} \mathrm{C}$.

Both aliphatic and indolic GSL biosynthesis are regulated by various genetic components. In Arabidopsis, such regulatory elements can repress GSL production such as SLIM1 (Sulfur Limitation1; MaruyamaNakashita et al., 2006), promote GSL production such as AtDof1.1 (DNA-binding-with-one finger; Skirycz et al., 2006) and subgroup 12 R2-R3 MYB transcription factors (Gigolashvili et al., 2009), or both positively and negatively regulate GSL production such as the nuclear-localized calmodulin-binding protein IQD1 (Levy et al., 2005). Two distinct clades of subgroup 12 R2-R3 MYB transcription factors regulate GSL biosynthesis and accumulation. Clade 1 members MYB34, $M Y B 51$, and MYB122 regulate indolic GSL biosynthesis, whereas clade 2 members $M Y B 28, M Y B 29$, and MYB76 regulate aliphatic GSL biosynthesis (Gigolashvili et al., 2009). Members within each clade appear to have distinct but overlapping roles. It has been suggested that MYB34, MYB122, and $M Y B 51$ positively regulate glucobrassicin, but only MYB51 positively regulates other GSLs such as methoxylated indolic glucosinolates (Gigolashvili et al., 2007). However, Malitsky and colleagues (2008) did not observe this trend and found that both MYB34 and $M Y B 51$ overexpression resulted in increases in 1-methoxyglucobrassicin.

MYB28 regulates both long- ( 6 to 8 carbon length) and short-chain (3 to 5 carbon length) aliphatic GSL biosynthesis and its overexpression can result in increased accumulation of indolic GSLs (Hirai et al., 2007). MYB29 and $M Y B 76$ also regulate GSL biosynthesis to a lesser degree, but only short-chain aliphatic GSL biosynthesis (Gigolashvili et al., 2008; Sønderby et al., 2007). MYB transcription factors may also regulate GSL biosynthesis in a tissue-specific manner with MYB34 and $M Y B 122$ being preferentially expressed in root tissues and MYB51 being preferentially expressed in leaf tissue (Gigolashvili et al., 2009). MYB28, MYB29, and MYB76 appear to be expressed to some degree in all tissues (Gigolashvili et al., 2009). In most cases, the expression pattern of MYB transcription factors correlates with expression of other GSL biosynthetic genes (Gigolashvili et al., 2009). No MYB transcription factors have been identified that specifically regulate aromatic GSL biosynthesis.

Using a comparative genomics approach, Zang and colleagues (2009) identified GSL biosynthetic and regulatory orthologs in 
Brassica rapa $(\mathrm{Br})$ with high sequence identity $(72 \%$ to $94 \%)$ to Arabidopsis thaliana GSL biosynthetic genes. In most cases, multiple copies of these genes were found in B. rapa (Zang et al., 2009). Two MYB transcription factor orthologs, MYB76 and $M Y B 122$, were absent or non-functional in B. rapa. BrMYB122-1 was found to be nonfunctional as a result of a deletion that resulted in a premature stop codon, whereas MYB76 was absent in B. rapa (Zang et al., 2009). The absence of MYB76 in B. rapa is interesting because $M Y B 76$ positively regulates methylsulfinylated short-chained aliphatic GLSs in Arabidopsis (Sønderby et al., 2010) and these GLSs are absent or in low concentrations in turnip (Carlson et al., 1987; Li et al., 2007; Smetanska et al., 2007). Although GSL-associated MYB orthologs from Arabipdopsis have been identified in B. rapa through comparative genomics (Zang et al., 2009), there is little data on the influence of these MYBs on GSL accumulation in B. rapa. It is possible that tissue-dependent changes in BrMYB transcription may result in tissue-dependent changes in GSL accumulation observed in turnips grown at different temperatures.

Genotype can be a major factor in determining baseline GSL concentration in plant tissues. Rosen and colleagues (2005) found that red cabbage had inherently higher GSL content than green cabbage. We previously found that roots of the red-skinned turnip cultivar Scarlet Queen have inherently higher GSL levels than purple-crown and whiteskinned turnip roots (Justen et al., 2011) Genotypes can also vary in terms of GSL enhancement by environmental factors (Fritz et al., 2010; Justen et al., 2011; Rosen et al., 2005). The objective of this experiment was to quantify shoot and root tissue GSL concentrations of two turnip cultivars, Just Right and Scarlet Queen, under three different temperature regimes representing the range of soil temperatures present in our previous plastic mulch experiment. We also sought to examine the relationship between temperature and GSL biosynthesis regulation by quantifying transcript levels of BrMYB orthologs in turnips grown at different temperatures. These MYB orthologs have been shown to regulate GSL biosynthesis in Arabidopsis.

\section{Materials and Methods}

Growth chamber conditions and plant materials. Turnip seeds (cv. Just Right; Jordan's Seed, Woodbury, MN, and cv. Scarlet Queen; Johnny's Seeds, Winslow, $\mathrm{ME}$ ) were grown in a soilless media (Sunshine Grow Mix LC-8; Sun Gro Horticulture, Bellevue, WA) in $10-\mathrm{cm}$ pots. The plants were grown for $42 \mathrm{~d}$ under three different air temperature treatments (Table 1) with a relative humidity of $75 \%$. The photoperiod was $14 \mathrm{~h}$ with a constant photosynthetically active radiation $(P A R)$ of $\approx 475 \mu \mathrm{mol} \cdot \mathrm{m}^{-2} \cdot \mathrm{s}^{-1}$ and a red:far-red ratio of 2.1. Plants were grown in a model GCW-15 growth chamber
(Environmental Growth Chambers, Chagrin Falls, $\mathrm{OH}$ ) fitted with metal halide bulbs. Plantlets were thinned to one plant per pot 1 week after seedling emergence. Plants were watered daily and fertilized weekly with 200 ppm nitrogen using 20-10-20 Peat-lite Water Soluble Fertilizer (Scotts Miracle-Gro Company, Marysville, OH).

All sampling was carried out between 0900 and $1100 \mathrm{HR}$. The experiment was replicated twice over time. JR and SQ plants were arranged in a completely randomized design of four replications within each growth chamber. Turnip plants were harvested 6 weeks after seeding and fresh weights were recorded after separation of shoots and roots. Turnip roots were 5 to $8 \mathrm{~cm}$ in diameter at the time of harvest. All samples were stored at $4{ }^{\circ} \mathrm{C}$ before processing for GSL quantification and were processed within $6 \mathrm{~h}$ after harvest.

Turnips used for gene expression analysis were seeded in a low- and high-temperature regime according as outlined in Table 1 . The light level was $\approx 500 \mu \mathrm{mol} \cdot \mathrm{m}^{-2} \cdot \mathrm{s}^{-1}$. JR and SQ were seeded in three biological replications at 10-d intervals. Samples from 6-weekold plants were harvested for gene expression analysis and immediately flash-frozen and stored at $-80{ }^{\circ} \mathrm{C}$ until RNA extraction. A subsample of this plant material was also used for GSL quantification according to the method that follows.

Glucosinolate quantification. Extraction and GSL quantification was performed as per Hecht et al. (2004) using modifications from Rosen et al. (2005) Briefly, a 50-g leaf sample or 100 -g root sample was incubated boiled in $300 \mathrm{~mL}$ of boiling water for $5 \mathrm{~min}$ to deactivate endogenous myrosinases. Boiled samples were macerated in a blender for $2 \mathrm{~min}$. A 40-mL aliquot of blended sample was homogenized using a BioSpec M133 Homogenizer (BioSpec Products, Inc., Bartlesville, OK) set at $12,000 \mathrm{rpm}$ for $2 \mathrm{~min}$ and then centrifuged for $10 \mathrm{~min}$ at $5000 \mathrm{~g}_{\mathrm{n}}, 4{ }^{\circ} \mathrm{C}$.

Desulfoglucosinolate (ds-GSL) extraction was performed using conditioned solid phase strong anion exchange (SAX) columns (Sigma-Aldrich, St. Louis, MO). Sinigrin (2-propenyl GSL; Sigma-Aldrich) was added to the conditioned SAX columns as an internal standard. To desulfate, samples were incubated with two units $\left(0.2 \mathrm{mg} \cdot \mathrm{mL}^{-1}\right)$ of sulfatase (aryl-sulfate sulfohydrolase; electrical conductivity 3.1.6.1; Sigma-Aldrich) on SAX columns for $\approx 15 \mathrm{~h}$ at room temperature $\left(\approx 21^{\circ} \mathrm{C}\right)$ then eluted with $3 \mathrm{~mL}$ water and the collected volume was determined by weight. Further washing of the columns yielded no additional ds-GSLs confirming complete elution. Eluent was stored at $-20{ }^{\circ} \mathrm{C}$ until high-performance liquid chromatography (HPLC) analysis.

HPLC analysis was performed on an Agilent 1200 Series Quaternary system (Agilent Technologies, Inc., Santa Clara, CA) monitoring absorbance at $\lambda=229 \mathrm{~nm}$ and using a Luna $\mathrm{C} 18,5-\mu \mathrm{m}, 250 \times 4.6-\mathrm{mm}$ column (Phenomenex, Torrance, CA) held at $30{ }^{\circ} \mathrm{C}$. A $50-\mu \mathrm{L}$ aliquot of the eluent was separated on the system with a flow rate of
Table 1. Temperature conditions used in growth chamber experiments. $^{\mathrm{z}}$

\begin{tabular}{lccc}
\hline & \multicolumn{3}{c}{ Temperature treatment $\left({ }^{\circ} \mathrm{C}\right)$} \\
\cline { 2 - 4 } Time & Low & Medium & High \\
\hline $0300 \mathrm{HR}$ & 15 & 17 & 21 \\
$1000 \mathrm{HR}$ & 19 & 24 & 28 \\
$1400 \mathrm{HR}$ & 22 & 27 & 34 \\
$2100 \mathrm{HR}$ & 19 & 24 & 28 \\
\hline
\end{tabular}

${ }^{\mathrm{z}}$ The photoperiod was from 0600 to $2000 \mathrm{HR}$.

$1.0 \mathrm{~mL} \cdot \mathrm{min}^{-1}$ using the following gradient: solvent $\mathrm{A}=$ water and $\mathrm{B}=$ acetonitrile; 0 to $2 \mathrm{~min}, 95 \% \mathrm{~A}, 5 \% \mathrm{~B} ; 2$ to $20 \mathrm{~min}, 85 \% \mathrm{~A}$, $15 \% \mathrm{~B} ; 20$ to $23 \mathrm{~min}, 53 \% \mathrm{~A}, 47 \% \mathrm{~B} ; 23$ to $30 \mathrm{~min}, 0 \% \mathrm{~A}, 100 \% \mathrm{~B}$; and 30 to $33 \mathrm{~min}$, $95 \%$ A, $5 \%$ B. Peaks were integrated using ChemStation for LC 3D Systems, Rev. B.04.01 software (Agilent Technologies, Inc.). GSL peak identities were confirmed by retention time and, as additional conformation, eluted compounds were analyzed using an Acquity Ultra Performance Liquid Chromatography-Mass Spectrometer (Waters Corporation, Milford, MA) using a C18 column, a water:acetontirile gradient, and negative electrospray ionization. ds-GSL concentrations were calculated using relative quantification with an internal standard (sinigrin) and previously published response factors (EU, 1990). ds-GSL concentrations are reported on a $\mu \mathrm{mol} / 100 \mathrm{~g}$ fresh weight basis.

Quantitative polymerase chain reaction analysis. Frozen turnip leaf and root tissues were ground in liquid nitrogen and then immediately used for RNA extraction with RNeasy Mini Kits and on-column DNAse digestion to remove genomic DNA contamination (Qiagen, Inc., Valencia, CA). Total RNA was quantified using a Qubit ${ }^{\text {TM }}$ fluorometer (Invitrogen Corp., Carlsbad, CA). Total RNA was reverse-transcribed into cDNA using the Super-Script III reverse transcriptase kit (Invitrogen Corp.). Quantitative polymerase chain reaction (PCR) and transcript analyses were performed using a Bio-Rad CFX96 ${ }^{\mathrm{TM}}$ Real-Time PCR detection system (Bio-Rad Laboratories, Hercules, CA).

PCR reactions contained $10 \mu \mathrm{L} \mathrm{iQ}^{\mathrm{TM}}$ SYBR Green Supermix (Bio-Rad Laboratories), $1 \mu \mathrm{L}$ each of $10 \mathrm{~mm}$ forward and reverse primer, $1 \mu \mathrm{L}$ cDNA template, and nucleasefree water to a final volume of $20 \mu \mathrm{L}$. A negative control (nuclease-free water) was included in each run. PCR primers were designed to amplify paralogs of a given gene family for BrMYB28, BrMYB29, BrMYB34, and BrMYB51 genes and BrActinl using a combination of Primerblast and MEGA sequence alignment tool (Version 4.1; Biodesign Institute, A240, Arizona State University, Tempe, AZ). Primers amplified a region containing at least one exon/intron split. Primer sequences are listed in Table S1. Sequence alignments between the sequenced PCR products for a given primer set and the intended gene to amplify are presented in Figure S1.

The PCR reaction was initiated with a denaturation step for $5 \mathrm{~min}$ at $94{ }^{\circ} \mathrm{C}$ followed by 40 cycles of denaturation $\left(94{ }^{\circ} \mathrm{C}\right.$ for $\left.30 \mathrm{~s}\right)$, 
annealing (depending on primer set, 50 to $54{ }^{\circ} \mathrm{C}$ for $30 \mathrm{~s}$ ), and extension $\left(72{ }^{\circ} \mathrm{C}\right.$ for $30 \mathrm{~s}$ ). Fluorescence was measured at the end of each extension step. The PCR reaction was ended with a final extension step for $7 \mathrm{~min}$ at $72{ }^{\circ} \mathrm{C}$ and then subjected to a melting curve analysis. The data were analyzed with CFX Manager software (Bio-Rad Laboratories). Transcript levels were normalized to Brassica rapa Actin 1 (BrACT1, Genbank accession number FJ969844). BrACT1 threshold cycle (Ct) values ranged between 19.1 and 22.6 across temperature treatments, tissues, and cultivars. A standard curve was generated for each primer set using a cDNA serial dilution to calculate PCR efficiencies used for relative transcript level analysis. Error bars shown represent the means \pm SE of three biological samples and three technical replicates (Fig. 1).

Statistical analysis. Data were analyzed with R 2.9.1 (R Foundation for Statistical Computing, Vienna, Austria). The significance of differences among treatments, cultivar, and experiments was assessed by a fixed-factor analysis of variance. Data are expressed as means. Mean values were considered significantly different at $P<0.05$ as determined by Tukey's honestly significant difference. Student's $t$ test was used to determine significant differences in transcript accumulation between samples.

Light measurements. PAR was measured with an Apogee Quantum Meter, Model QMSW-SS (Apogee Instruments, Inc., Logan, UT), and red:far-red ratios were measured with a Skye red-far-red meter (Skye Instruments, Ltd., Powys, U.K.).

\section{Results and Discussion}

Fresh weight. Cultivar-by-temperature interactions and temperature-by-experiment interactions were significant for fresh weight and several individual GSL concentrations; therefore, data will be presented separately for experiments, cultivars, and tissue types (Tables 2 and 3). Temperature was a significant factor influencing root and shoot fresh weight in both JR and SQ (Table 2). In shoots, there was a significant reduction in shoot fresh weight in low- and mediumtemperature treatments when compared with the high-temperature treatments in Expt. 2 but not Expt. 1 (Table 2). The opposite was observed in roots, in which substantial decreases in root biomass occurred in hightemperature treatments when compared with low- and medium-temperature treatments (Table 2). The influence of temperature on root yield is important because biomass accumulation has been inversely correlated with GSL concentration (Antonious et al., 1996; Radovich et al., 2005; Rosen et al., 2005). Thus, increasing temperatures may result in higher GSL concentration yet lead to less GSL yield per plant. Despite the differences in fresh weight observed in our experiment, GSL concentrations per root were still $\approx 30 \%$ and $15 \%$ higher in JR and SQ roots, respectively, grown at the high temperature than at the low temperature (Table 3).

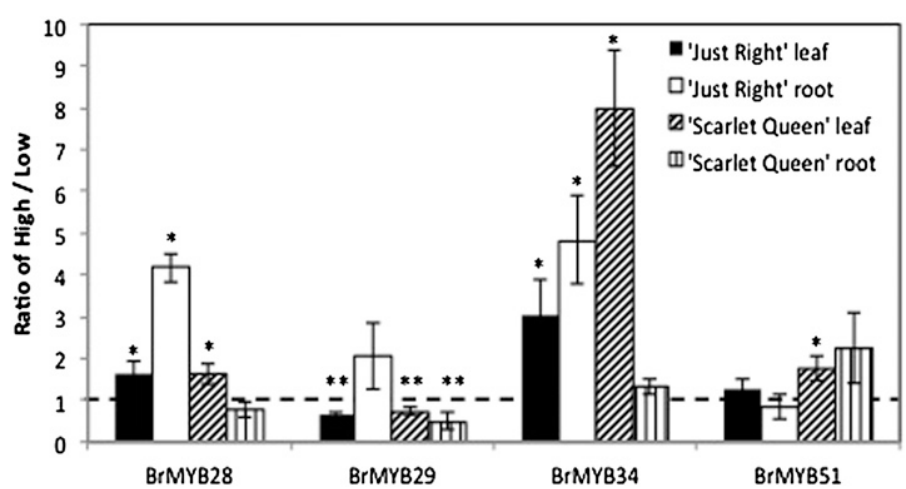

Fig. 1. Transcript levels of turnip glucosinolate regulatory genes determined by real-time polymerase chain reaction. Ratio values are the fold difference between the normalized transcript levels (relative to actin) in 'Just Right' and 'Scarlet Queen' turnip tissues grown in high- and low-temperature regimes. Error bars are SE. **,* represent ratios significantly less than 1 or greater than 1 , respectively, at $P<0.07$.

Table 2. Fresh weight of 'Just Right' and 'Scarlet Queen' turnip shoots and roots across experiments and temperature treatments.

\begin{tabular}{|c|c|c|c|c|}
\hline \multirow[b]{3}{*}{ Cultivar } & \multirow[b]{3}{*}{ Tissue } & \multirow[b]{3}{*}{ Temperature treatment } & \multicolumn{2}{|c|}{ Fresh wt $(g)^{z, y}$} \\
\hline & & & \multicolumn{2}{|c|}{ Expt. } \\
\hline & & & 1 & 2 \\
\hline \multirow[t]{6}{*}{ Just Right } & Shoot & Low & $122 \mathrm{a}$ & $110 \mathrm{c}$ \\
\hline & & Medium & $122 \mathrm{a}$ & $168 \mathrm{~b}$ \\
\hline & & High & $133 \mathrm{a}$ & $224 \mathrm{a}$ \\
\hline & Root & Low & $216 \mathrm{~b}$ & $320 \mathrm{~b}$ \\
\hline & & Medium & $272 \mathrm{a}$ & $370 \mathrm{a}$ \\
\hline & & High & $108 \mathrm{c}$ & $240 \mathrm{c}$ \\
\hline \multirow[t]{6}{*}{ Scarlet Queen } & Shoot & Low & $97 \mathrm{a}$ & $87 \mathrm{~b}$ \\
\hline & & Medium & $83 \mathrm{a}$ & $117 \mathrm{~b}$ \\
\hline & & High & $90 \mathrm{a}$ & $183 \mathrm{a}$ \\
\hline & Root & Low & $206 \mathrm{a}$ & $309 \mathrm{a}$ \\
\hline & & Medium & $229 \mathrm{a}$ & $348 \mathrm{a}$ \\
\hline & & High & $94 \mathrm{~b}$ & $229 \mathrm{~b}$ \\
\hline
\end{tabular}

${ }^{\mathrm{z}}$ Means with different letters within each column, cultivar, and tissue type are significantly different at $P \leq$ 0.05 as determined by Tukey's honestly significant difference.

${ }^{y}$ Fresh weight $(\mathrm{g})$ determined from two turnip plants at the time of harvest.

Glucosinolate concentration. Temperature had a significant effect on total GSL concentration (TTL) in shoot and root tissue of both JR and SQ (Table 3). In all cases, shoot and root TTL were always significantly lower in the low- and medium-temperature treatments than the high temperature (Table 3 ). This temperature-induced increase in GSLs resulted in an $\approx 180 \%$ increase in TTL concentration in roots and shoots grown in the high-temperature treatment when compared with the low-temperature treatment. Although high temperatures significantly reduced root yield, the high-temperature treatment still resulted in increased TTL per plant when compared with the low-temperature treatment ( 356 vs. $256 \mu \mathrm{mol} /$ root for JR and 428 vs. $369 \mu \mathrm{mol} /$ root for SQ) suggesting that the increase in GSL concentrations resulting from high temperatures was not strictly the result of a dilution effect.

Cultivars and tissues varied in individual glucosinolate concentrations and profiles. In shoot tissue, two GSLs, GNP (3-butenyl GSL) and glucobrassicanapin (GBN; 4-pentenyl GSL), comprised $\approx 95 \%$ of the total glucosinolate concentration. In root tissue, five GSLs, progoitrin (PRO; 2-hydroxy-3-butenyl GSL), GNP, GBN, 1-MGB, and GNS, comprised $\approx 90 \%$ of the total glucosinolate concentration. Therefore, the effect of temperature on individual GSLs is discussed in detail only for these five GSLs. In JR root tissue, GNP was the dominant GSL, comprising $\approx 50 \%$ of TTL across temperature treatments (Table 3 ). In SQ roots, the dominant GSL was dependent on the temperature treatment with GNS being the predominant GSL ( $36 \%$ of TTL) in low temperatures and PRO being the dominant GSL (32\% of TTL) in high temperature. GSL profiles for JR and SQ are similar to those observed in our colored plastic mulch experiments; however, absolute GSL concentrations were much lower in the field experiments (Justen et al., 2011) than the growth chamber experiments $(\approx 35 \%$ and $\approx 60 \%$ less for shoots and roots, respectively). Our results for SQ roots are also consistent with previous data establishing GNS and PRO to be the primary GSLs in turnip root tissue (Zhang et al., 2008) and GNP to be the primary GSL in turnip shoot tissue (Carlson et al., 1987; Smetanska et al., 2007).

The effect of temperature on individual GSLs varied depending on the cultivar, tissue type, and experiment (Table 3). Differences in GSL concentrations between experiments could be the result of the dynamic nature of 


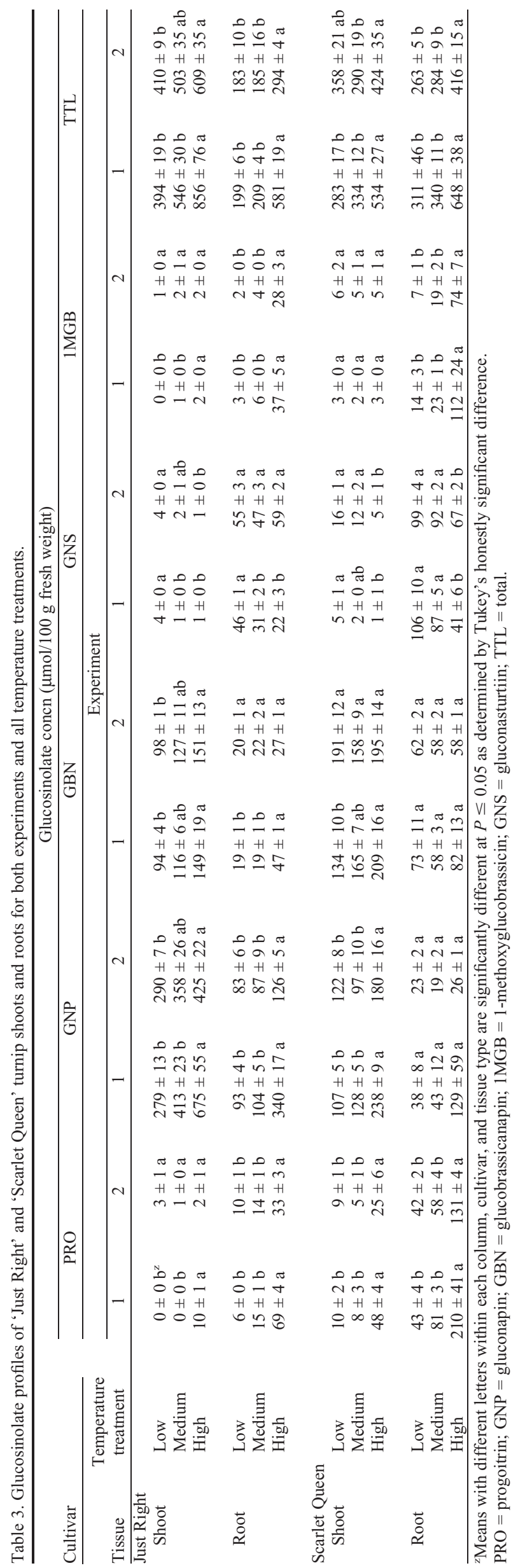

GSLs as has been observed in previous studies (Justen et al., 2011; Rosen et al., 2005). In JR shoots, GNP was the GSL most influenced by temperature treatment with the high-temperature treatment increasing GNP by $182 \%(266 \mu \mathrm{mol} / 100 \mathrm{~g}$ fresh weight $)$ and $140 \%(165 \mu \mathrm{mol} / 100 \mathrm{~g}$ fresh weight $)$ when compared with low and medium treatments, respectively. A similar trend was observed in JR roots and SQ shoots but not SQ roots. This increase in GNP is similar to that found by Charron and colleagues who saw GNP double in leaves and stems of $B$. oleracea plants grown at elevated temperatures (Charron and Sams, 2004). In JR shoots, GBN also increased a similar percentage as GNP with temperature; however, because GBN is lower in JR shoots, the absolute increases were not as large (54 and $29 \mu \mathrm{mol} / 100 \mathrm{~g}$ fresh weight increase when compared with low- and medium-temperature treatments, respectively). PRO was consistently increased in SQ shoots in high-temperature treatments but not in JR shoots. GNS concentration decreased with increased temperature in JR and SQ shoots. High temperature-grown JR shoots had $75 \%$ less GNS than those grown at low temperature; however, GNS is present at inherently low concentrations in turnip shoot tissues; thus, these decreases had minimal effects on the profile. Shoot $1 \mathrm{MGB}$ concentration was not significantly altered by temperature in most instances.

Root tissue GSLs were also significantly impacted by temperature (Table 3 ). Although the high treatment resulted in dramatic changes in GSL content, the medium treatment led to minimal changes in most GSLs when compared with the low-temperature treatment. In the high treatment, 1MGB increased by $30 \mu \mathrm{mol}$ in JR roots and $82 \mu \mathrm{mol} /$ $100 \mathrm{~g}$ fresh weight in SQ roots, respectively. This amounts to an $1100 \%$ and $850 \%$ increase in 1MGB for JR and SQ, respectively. This temperature-dependent increase in 1MGB in root tissue was also demonstrated by Charron and Sams (2004) who reported a 3-fold increase in $1 \mathrm{MGB}$ in $B$. oleracea roots grown at $32{ }^{\circ} \mathrm{C}$ compared with those grown at $12{ }^{\circ} \mathrm{C}$. The fact that $1 \mathrm{MGB}$ can be manipulated so drastically with environmental conditions speaks greatly to the role of cultural practices in yielding vegetables with great nutraceutical potential. The bioactive hydrolysis product of 1MGB (N-methoxyindole-3-carbinol) has been shown to have chemopreventive properties exceeding that of indole-3-carbinol, the bioactive hydrolysis product of glucobrassicin (Neave et al., 2005). Temperaturedependent increases in GNP, GBN, and PRO were also observed in root tissues, but these varied with cultivar. In JR roots, GNP and GBN values were increased by 265\% (145 $\mu \mathrm{mol} / 100 \mathrm{~g}$ fresh weight) and $195 \%$ (18 $\mu \mathrm{mol} / 100 \mathrm{~g}$ fresh weight), respectively, between the high-temperature treatment and the low-temperature treatment. In SQ roots, temperature did not have a significant effect on GNP and GBN concentrations. However, PRO in JR and SQ roots was increased by $600 \%$ (43 $\mu \mathrm{mol} / 100 \mathrm{~g}$ fresh weight) and 398\% 
(128 $\mu \mathrm{mol} / 100 \mathrm{~g}$ fresh weight), respectively, between the high-temperature treatment and the low-temperature treatment.

In root tissues, GNS concentration decreased in the high-temperature treatment. The extent of temperature-dependent GNS changes varied with cultivar and experiment. In JR roots, GNS concentrations were significantly decreased in the low-temperature treatment in Expt. 1 but not Expt. 2. In SQ roots, GNS concentrations were consistently reduced in high-temperature treatments. This decrease in GNS is consistent with previous data showing an inverse relationship between growth temperature and GNS content in watercress (Engelen-Eigles et al., 2006). The variability in temperature-induced GNS concentrations between cultivars is consistent with previous work on cabbage varieties in which GSL enhancement from varying fertility levels and jasmonic acid applications has been cultivar-dependent (Fritz et al., 2010; Rosen et al., 2005). It is possible that the glucosinolate profiles of some genotypes are more influenced by environmental treatments than others.

Glucosinolate regulatory gene transcript levels. One possible reason for the increases we observed in GSL accumulation at increased temperature may be the result of changes in GSL biosynthesis. Previous research in Arabidopsis has shown that MYB transcription factors play a significant role in regulating GSL biosynthesis (Gigolashvili et al., 2009; Hirai et al., 2007). Using primers based on B. rapa GSL MYB orthologs, we performed quantitative PCR to examine the relationship between GSL regulatory gene transcript levels and temperature and to determine if these transcript levels are associated with the GSL phenotype we observed with different temperature treatments.

We examined the regulatory genes BrMYB28, BrMYB29, BrMYB34, and BrMYB51 in root and shoot tissues of two turnip cultivars grown at a high- and low-temperature treatment. Our results indicate that GSL MYB transcript levels are influenced by temperature and are associated with changes in GSL accumulation in some cultivars and tissues (Fig. 1). In Arabidopsis, MYB28 and MBY29, members of a distinct clade of subgroup 12 R2R3 MYB transcription factors, have been shown to positively regulate aliphatic GSL production with MYB28 having the stronger regulatory effect of the two MYBs (Hirai et al., 2007). Normalized BrMYB28 transcript levels were higher in high temperature than low temperature-treated plants (ratio greater than 1) in all tissues but SQ roots. BrMYB28 transcript levels agree with GSL concentration data because there was a significant increase in the aliphatic GSLs, GNP, and GBN in all tissues grown in the high-temperature treatment except SQ roots (Table 3). In SQ roots, GNP or GBN were not elevated with temperature treatment (Table 3) and BrMYB28 transcript levels in SQ root tissue did not increase with temperature (Fig. 1). Normalized BrMYB29 transcripts levels followed a similar pattern as $\operatorname{BrMYB2} 8$ across the tissues and cultivars with the ratio being highest in JR roots and lowest in SQ roots. Unlike BrMYB28, BrMYB29 levels appeared to be repressed by high temperatures as indicated by the ratio less than 1 for all samples except JR roots; thus, there does not appear to be a consistent association between aliphatic GSL accumulation and BrMYB29 transcript levels. A similar discrepancy between MYB29 expression and GSL content was also observed in Arabidopsis in which MYB29 gene knockdown did not result in apparent changes in GSL content (Hirai et al., 2007). It is interesting that the aliphatic GSL, PRO, was significantly elevated at high temperatures in SQ root tissues, but neither BrMYB28 nor BrMYB29 transcript levels were increased in these tissues in the high-temperature treatment. These results suggest that PRO may be under different regulatory control than GNP and GBN.

Indolic GSL accumulation has been shown to be under the control of coordinated activities of MYB34 and MYB51 (Gigolashvili et al., 2009). These two transcription factors belong to a separate clade of subfamily 12 R2R3-MYB transcription factors than MYB28 and MYB29. In our study, there was not a clear relationship between indolic GSL concentration and BrMYB34 transcript levels. This may be because turnips only accumulate 1MGB, a methoxylated indolic GSL. Previous investigations have shown an inconsistent relationship between methoxylated indolic GSL concentration and BrMYB34 transcript levels (Gigolashvili et al., 2007; Malitsky et al., 2008). Similar to BrMYB28, BrMYB34 transcript levels were significantly elevated with temperature treatments in all tissues except SQ roots. These results suggest a positive association between TTL concentration in JR roots and shoots and SQ shoots and BrMYB34 transcript levels. The similarity of transcript level patterns between BrMYB2 8 and BrMYB34, but not between BrMYB34 and $B r M Y B 51$, supports previous research that suggests a positive correlation between expression levels of MYB34 and members of the $M Y B 28$ clade but a negative relationship between MYB34 and MYB51 expression in Arabidopsis (Malitsky et al., 2008). MYB51 has been suggested to positively regulate methoxlyated indolic GSL biosynthesis in Arabidopsis (Gigolashvili et al., 2007). Although we did observe a significant increase in 1MGB with the high-temperature treatment in roots but not shoots (Table 3 ), these GSL data were not associated with BrMYB51 transcript levels because there was no significant change in BrMYB51 transcript levels in roots between the two temperature treatments (Fig. 1).

Previous investigations have suggested that crosstalk occurs between the two clades of GSL MYBs so that relative changes in expression of ATRl-like clade members (MYB34 and MYB51) are inversely correlated with relative changes in expression of MYB28-like clade members (MYB28 and MYB29) in Arabidopsis (Gigolashvili et al., 2009; Malitsky et al., 2008). Our results partially support this claim because increases in relative transcript levels of $B r M Y B 34$, a member of the ATR1-like clade, corresponded in most cases with decreases in relative transcript levels of BrMYB29, a member of the MYB28-like clade.

\section{Conclusion}

Temperature significantly impacted GSL accumulation with increases in temperature resulting in increased TTL, aliphatic and indolic GSL concentrations, and decreased GNS concentrations. GSL regulatory gene expression was differentially influenced by temperature treatment depending on the tissue type and cultivar with some positive associations existing between temperature and GSL regulatory transcript levels. These results further emphasize the importance of temperature on GSL accumulation. The fact that some BrMYB transcript levels were both positively associated with temperature and GSL concentrations in some turnip tissues suggests that temperature may play a role in regulating GSL biosynthesis in B. rapa.

\section{Literature Cited}

Antonious, G.F., M.J. Kasperbauer, and M.E Byers. 1996. Light reflected from colored mulches to growing turnip leaves affects glucosinolate and sugar contents of edible roots. Photochem. Photobiol. 64:605-610.

Carlson, D.G., M.E. Daxenbichler, H.L. Tookey, W.F. Kwolek, C.B. Hill, and P.H. Williams 1987. Glucosinolates in turnip tops and roots: Cultivars grown for greens and/or roots. J. Amer. Soc. Hort. Sci. 112:179-183.

Charron, C.S. and C.E. Sams. 2004. Glucosinolate content and myrosinase activity in rapidcycling Brassica oleracea grown in a controlled environment. J. Amer. Soc. Hort. Sci. 129:321330.

Engelen-Eigles, G., G. Holden, J.D. Cohen, and G Gardner. 2006. The effect of temperature, photoperiod, and light quality on gluconasturtiin concentration in watercress (Nasturtium officinale R. br.). J. Agr. Food Chem. 54:328334.

EU. 1990. Off. J Eur Commun. L 170:03.07. p. 27-34.

Fritz, V.A., V.L. Justen, A.M. Bode, T. Schuster, and M. Wang. 2010. Glucosinolate enhancement in cabbage induced by jasmonic acid application. HortScience 45:1188-1191.

Gigolashvili, T., B. Berger, and U. Flügge. 2008 HAG2/MYB76 and HAG3/MYB29 exert a specific and coordinated control on the regulation of aliphatic glucosinolate biosynthesis in $\mathrm{Ara}$ bidopsis thaliana. New Phytol. 177:627-642.

Gigolashvili, T., B. Berger, and U. Flügge. 2009. Specific and coordinated control of indolic and aliphatic glucosinolate biosynthesis by R2R3MYB transcription factors in Arabidopsis thaliana. Phytochem. Rev. 8:3-13.

Gigolashvili, T., R. Yatusevich, B. Berger, C. Müller, and U. Flügge. 2007. The R2R3-MYB transcription factor HAG1/MYB28 is a regulator of methionine-derived glucosinolate biosynthesis in Arabidopsis thaliana. Plant J. 51:247-261.

Hecht, S.S. 2000. Inhibition of carcinogenesis by isothiocyanates. Drug Metab. Rev. 32:395-411.

Hecht, S.S., S.G. Carmella, P.M.J. Kenney, S.H. Low, K. Arakawa, and M.C. Yu. 2004. Effects of cruciferous vegetable consumption on urinary metabolites of the tobacco-specific lung carcinogen 4-(methyinitrosamino)-1-(3-pyridyl)1-butanone in Singapore Chinese. Cancer Epidemiol. Biomarkers Prev. 13:997-1004. 
Hirai, M.Y., K. Sugiyama, Y. Sawada, T. Tohge, T. Obayashi, A. Suzuki, R. Araki, N. Sakurai, H. Suzuki, K. Aoki, H. Goda, O.I. Nishizawa, D. Shibata, and K. Saito. 2007. Omics-based identification of Arabidopsis Myb transcription factors regulating aliphatic glucosinolate biosynthesis. Proc. Natl. Acad. Sci. USA 104: 6478-6483.

Justen, V.L., J.D. Cohen, G. Gardner, and V.A. Fritz. 2011. Seasonal variation in glucosinolate accumulation in turnip cultivars grown with colored plastic mulches. HortScience 46:1608 1614.

Levy, M., Q. Wang, R. Kaspi, M. Parrella, and S. Abel. 2005. Arabidopsis IQD1, a novel calmodulin-binding nuclear protein, stimulates glucosinolate accumulation and plant defense. Plant J. 43:79-96.

Li, S., I. Schonhof, A. Krumbein, L. Li, H. Stützel, and M. Schreiner. 2007. Glucosinolate concentration in turnip (Brassica rapa ssp. rapifera L.) roots as affected by nitrogen and sulfur supply. J. Agr. Food Chem. 55:84528457.

Malitsky, S., E. Blum, H. Less, I. Venger, M. Elbaz, S. Morin, Y. Eshed, and A. Aharoni. 2008. The transcript and metabolite networks affected by the two clades of Arabidopsis glucosinolate biosynthesis regulators. Plant Physiol. 148:2021-2049.
Maruyama-Nakashita, A., Y. Nakamura, T. Tohge, K. Saito, and H. Takahashi. 2006. Arabidopsis SLIM1 is a central transcriptional regulator of plant sulfur response and metabolism. Plant Cell 18:3235-3251.

Neave, A.S., S.M. Sarup, M. Seidelin, F. Duus, and O. Vang. 2005. Characterization of the $\mathrm{N}$-methoxyindole-3-carbinol (NI3C)-induced cell cycle arrest in human colon cancer cell lines. Toxicol. Sci. 83:126-135.

Radovich, T.J.K., M.D. Kleinhenz, J.G. Streeter, A.R. Miller, and J.C. Scheerens. 2005. Planting date affects total glucosinolate concentrations in six commercial cabbage cultivars. HortScience 40:106-110.

Rosen, C.J., V.A. Fritz, G.M. Gardner, S.S. Hecht, S.G. Carmella, and P.M. Kenney. 2005. Cabbage yield and glucosinolate concentrations as affected by nitrogen and sulfur fertility. HortScience 40:1493-1498.

Skirycz, A., M. Reichelt, M. Burow, C. Birkemeyer, J. Rolcik, J. Kopka, M.I. Zanor, J. Gershenzon, M. Strnad, J. Szopa, B. Mueller-Roeber, and I. Witt. 2006. DOF transcription factor AtDof1.1 (OBP2) is part of a regulatory network controlling glucosinolate biosynthesis in arabidopsis. Plant J. 47:10-24.

Smetanska, I., A. Krumbein, M. Schreiner, and D. Knorr. 2007. Influence of salicylic acid and methyl jasmonate on glucosinolate levels in turnip. J. Hort. Sci. Biotechnol. 82:690694.

Sønderby, I., B. Hansen, N. Bjarnholt, C. Ticconi, B. Halkier, and D. Kliebenstein. 2007. A systems biology approach identifies a R2R3 MYB gene subfamily with distinct and overlapping functions in regulation of aliphatic glucosinolates. PLoS One 2:E1322.

Sønderby, I.E., M. Burow, H.C. Rowe, D.J. Kliebenstein, and B.A. Halkier. 2010. A complex interplay of three R2R3 MYB transcription factors determines the profile of aliphatic glucosinolates in Arabidopsis. Plant Physiol. 153:348-363

Talalay, P. and J.W. Fahey. 2001. Phytochemicals from cruciferous plants protect against cancer by modulating carcinogen metabolism. J. Nutr. 131:3027S-3033S.

Zang, Y.-X., H. Kim, J. Kim, M.-H. Lim, M. Jin, S. Lee, S.-J. Kwon, S.-I. Lee, J.K. Hong, T.-H. Park, J.-H. Mun, Y.-J. Seol, S.-B. Hong, and B.-S. Park. 2009. Genome-wide identification of glucosinolate synthesis genes in Brassica rapa. FEBS J. 276:3559-3574

Zhang, H., I. Schonhof, A. Krumbein, B. Gutezeit, L. Li, H. Stützel, and M. Schreiner. 2008. Water supply and growing season influence glucosinolate concentration and composition in turnip root (Brassica rapa ssp. rapifera $\mathrm{L}$.). J. Plant Nutr. Soil Sci. 171:255-265. 


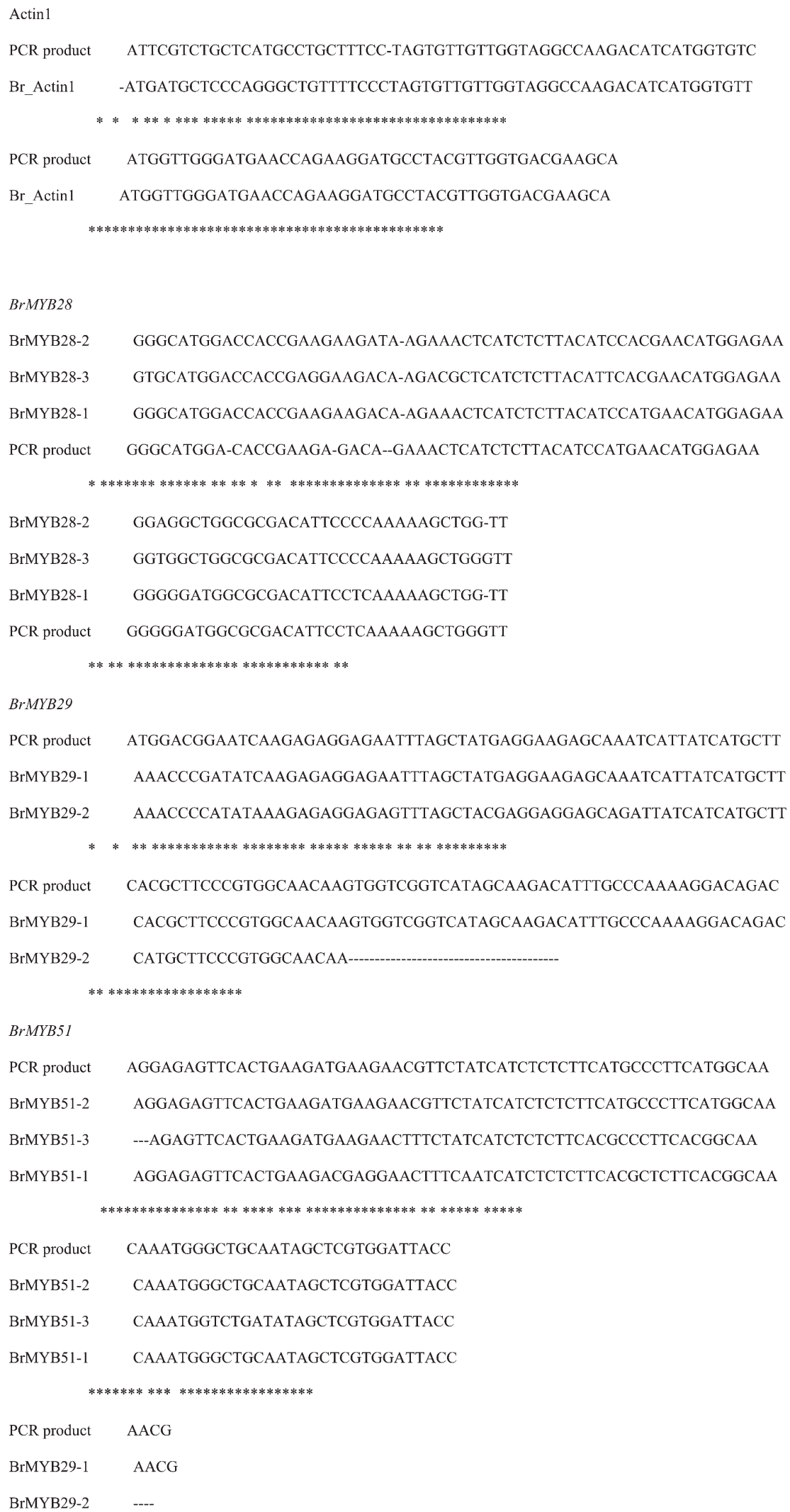

Fig. S1. Clustal W alignments between published Brassica rapa gene sequences and sequenced polymerase chain reaction products of turnip tissues amplified by primers listed in Table S1. 
Table S1. Forward and reverse primers used in real-time polymerase chain reaction analyses.

\begin{tabular}{|c|c|c|c|}
\hline \multicolumn{4}{|l|}{$\begin{array}{l}\text { Gene } \\
\text { identifier } \\
\text { (Genbank) }\end{array}$} \\
\hline FJ584287 & BrMYB28-1 & AAGAAAGCCATGTTGTGTCG & TTTCCACACCTTTTCAACCC \\
\hline FJ584288 & BrMYB28-2 & & \\
\hline FJ584290 & BrMYB29-1 & AGTTGTAGATTGCGATGGGC & CGTTGTCTGTCCTTTTGGGC \\
\hline FJ584291 & BrMYB29-2 & & \\
\hline FJ584294 & BrMYB34-2 & & \\
\hline FJ584295 & BrMYB34-3 & & \\
\hline FJ584296 & BrMYB51-1 & AGATGTGGCAAAAGCTGCAGA & GGTAATCCACGAGCTATTGCA \\
\hline FJ584297 & BrMYB51-2 & & \\
\hline FJ584298 & BrMYB51-3 & & \\
\hline FJ584299 & BrMYB51-4 & & \\
\hline
\end{tabular}

